\title{
Biorthogonal Quincunx Coifman Wavelets
}

\author{
Dong Wei, Brian L. Evans, and Alan C. Bovik* \\ Laboratory for Vision Systems \\ Department of Electrical and Computer Engineering \\ The University of Texas at Austin, Austin, TX 78712-1084, U.S.A. \\ E-mail: $\{$ dwei, bevans, bovik\}@ece.utexas . edu
}

\begin{abstract}
We define and construct a new family of compactly supported, nonseparable two-dimensional wavelets, "biorthogonal quincunx Coifman wavelets" (BQCWs), from their one-dimensional counterparts using the $M c$ Clellan transformation. The resulting filter banks possess many interesting properties such as perfect reconstruction, vanishing moments, symmetry, diamondshaped passbands, and dyadic fractional filter coefficients. We derive explicit formulas for the frequency responses of these filter banks. Both the analysis and synthesis lowpass filters converge to an ideal diamondshaped halfband lowpass filter as the order of the corresponding $B Q C W$ system tends to infinity. Hence, they are promising in image and multidimensional signal processing applications. In addition, the synthesis scaling function in a $B Q C W$ system of any order is interpolating (or cardinal), which has been known as a desired merit in numerical analysis.
\end{abstract}

\section{Introduction}

During the past decade, the theory of wavelets has established itself firmly as one of the most successful methods for many signal processing applications, such as image coding, noise reduction, and singularity detection, to name a few, primarily because wavelet expansions are more appropriate than Fourier series to represent the local behavior of non-stationary signals.

However, most of these developments have concentrated on one-dimensional (1D) signals and the multidimensional (MD) case was handled via the tensor product to yield separable systems [1]. Using separable wavelets preserves some properties of $1 \mathrm{D}$ wavelets, such as finite support, perfect reconstruction (PR), orthonormality, symmetry, and regularity, and often

${ }^{*}$ This work was supported in part by a grant from Southwestern Bell Technology Resources, Inc., NSF CAREER Award under Grant MIP-9702707, and a UT-Austin Summer Research Assignment Grant. leads to simple implementations and low computational complexity. However, it imposes a severe limitation on the resulting MD wavelet bases in the sense that it gives a particular importance to the vertical and horizontal directions. Therefore, when dealing with MD signals, true MD processing (allowing both nonseparable sampling and filtering) is more appropriate. Though nonseparable wavelet bases suffer from higher computational complexity, they offer more flexibility (e.g. near-isotropic processing) in multiresolution analysis, more degrees of freedom in design, better adaption to the human visual system, and consequently better performance. In particular, nonseparable two-dimensional (2D) wavelet bases are of great importance in image processing applications. On the other hand, since orthogonality and symmetry are a pair of conflicting properties for compactly supported wavelets, biorthogonal symmetric wavelet bases whose associated filter banks (FBs) possess linear phase are the most widely used in practice. Linear phase is often a very desirable property in image processing.

The construction of nonseparable $2 \mathrm{D}$ wavelets has been a challenging problem because the fundamental method used in the design of $1 \mathrm{D}$ wavelets, spectral factorization, cannot be extended to construct $2 \mathrm{D}$ nonseparable wavelets, because $2 \mathrm{D}$ polynomials cannot always be factored. The McClellan transformation [2] has been recognized as a useful tool to construct quincunx wavelets from 1D prototype FBs [3], [4]. The goal of the paper is to construct a novel class of compactly supported biorthogonal quincunx wavelets using the McClellan transformation.

The following notation will be used in the paper. Boldfaced lowercase and uppercase letters denotes 2D vectors and matrices, respectively. The impulse response and the frequency response of a filter are denoted, respectively, by lowercase and uppercase letters. Due to space limitations, the proofs of the theorems presented in this paper are not included, but will 
be given elsewhere.

\section{One-dimensional biorthogonal Coif- man wavelets}

Recently, the biorthogonal Coifman wavelet (BCW) has been constructed independently in [5] and [6]. The dual lowpass filters in an even-ordered BCW system are symmetric. Hence, their frequency responses possess a zero phase. The frequency response of the $m$ thorder synthesis filter is given by

$$
\begin{aligned}
H_{m}(\omega)= & \left(\frac{1+\cos \omega}{2}\right)^{\frac{m}{2}} \\
& \times \sum_{l=0}^{m / 2-1}\left(\begin{array}{c}
\frac{m}{2}-1+l \\
l
\end{array}\right)\left(\frac{1-\cos \omega}{2}\right)^{l}
\end{aligned}
$$

if $m$ is even. It possesses the same number of zeroes at DC and the aliasing frequency $\pi$. The frequency response of the analysis filter of order $\left(m, m^{\prime}\right)$ can be expressed as

$$
\widetilde{H}_{m, m^{\prime}}(\omega)=2 H_{m^{\prime}}(\omega)+H_{m}(\omega)-2 H_{m^{\prime}}(\omega) H_{m}(\omega)
$$

if $m$ and $m^{\prime}$ are even and $m \geq m^{\prime}$. For the case $m<m^{\prime}, \widetilde{H}_{m, m^{\prime}}(\omega)$ uniquely exists but possesses a complicated analytic form.

It has been shown in [5] and [6] that the BCW systems have many useful properties including (i) dyadic fractional filter coefficients, which yield fast implementations (only additions and binary shifts are needed); (ii) excellent potential for image compression, which turns out to be superior to the biorthogonal spline wavelet (BSW) systems and competitive to the widely used FBI $(9,7)$-tap FB proposed in [7]; and (iii) one of the two associated scaling function is interpolating (or cardinal) so that the wavelet expansion coefficients can be approximated by function samples with very high accuracy, which has been known as a desired merit in numerical analysis.

\section{Biorthogonal quincunx Coifman wavelets}

\subsection{Definition and construction}

When dealing with MD wavelet bases, the change in resolution and sampling rate is given by an integer dilation matrix $\boldsymbol{D}$. For quincunx wavelets, it is required that $\boldsymbol{D} \boldsymbol{n}, \boldsymbol{n} \in \mathbb{Z}^{2}$, is a quincunx sublattice of $\mathbb{Z}^{2},|\operatorname{det} \boldsymbol{D}|=2$, and the two eigenvalues of $\boldsymbol{D}$ have magnitude strictly greater than unity so that there is indeed a dilation in each dimension [3], [4]. The following matrices are two typical choices:

$$
\boldsymbol{D}_{1} \triangleq\left[\begin{array}{rr}
1 & 1 \\
1 & -1
\end{array}\right] \quad \text { or } \quad \boldsymbol{D}_{2} \triangleq\left[\begin{array}{rr}
1 & -1 \\
1 & 1
\end{array}\right] \text {. }
$$

Figure 1 and Figure 2 illustrate the block diagrams of two-channel iterative analysis and synthesis FBs, respectively, where $\widetilde{h}$ and $\widetilde{g}$ are respectively analysis lowpass and highpass filters, and $h$ and $g$ are respectively synthesis lowpass and highpass filters. If $\boldsymbol{D}=2$, then it reduces to a $1 \mathrm{D} \mathrm{FB}$; if $\boldsymbol{D}=\boldsymbol{D}_{1}$ or $\boldsymbol{D}=\boldsymbol{D}_{2}$, then it represents a quincunx FB, in which the highpass filters $g(\boldsymbol{n})$ and $\widetilde{g}(\boldsymbol{n})$ are given by (see [3], [4])

$$
\begin{aligned}
G(\boldsymbol{\omega}) & =e^{-j\left(\omega_{1}+\omega_{2}\right)} \widetilde{H}^{*}(\boldsymbol{\omega}+\boldsymbol{\pi}), \\
\widetilde{G}(\boldsymbol{\omega}) & =e^{-j\left(\omega_{1}+\omega_{2}\right)} H^{*}(\boldsymbol{\omega}+\boldsymbol{\pi}) .
\end{aligned}
$$

The 2D PR condition can be expressed as, $\forall \boldsymbol{\omega} \in \mathbb{R}^{2}$,

$$
H(\boldsymbol{\omega}) \widetilde{H}^{*}(\boldsymbol{\omega})+H(\boldsymbol{\omega}+\boldsymbol{\pi}) \widetilde{H}^{*}(\boldsymbol{\omega}+\boldsymbol{\pi})=1
$$

with $h$ and $\widetilde{h}$ satisfying the admissibility conditions: $H(\mathbf{0})=\widetilde{H}(\mathbf{0})=1, H(\boldsymbol{\pi})=\widetilde{H}(\boldsymbol{\pi})=0$, where $\mathbf{0}=$ $[0,0]^{T}$ and $\pi=[\pi, \pi]^{T}$.

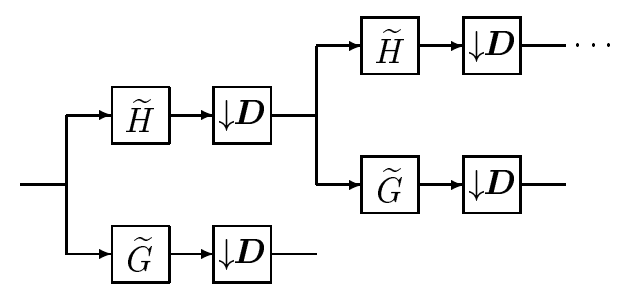

Figure 1: A two-channel iterative analysis FB

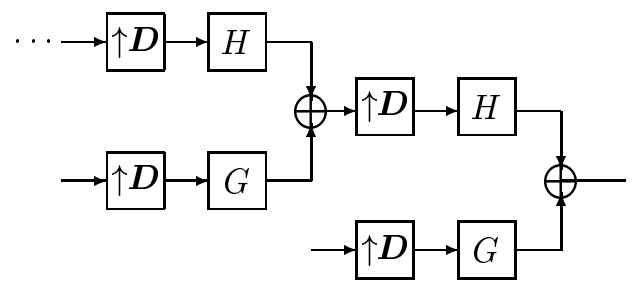

Figure 2: A two-channel iterative synthesis FB

We propose the following theorem to define a novel class of biorthogonal quincunx wavelets.

Theorem 1. The following three sets of conditions are equivalent, and each one can serve as a definition of a biorthogonal quincunx Coifman wavelet (BQCW) of order $L$.

1. All moments up to order $(L-1)$ of the scaling function and the wavelet vanish, that is

$$
\int \boldsymbol{t}^{\boldsymbol{l}} \phi(\boldsymbol{t}) d \boldsymbol{t}=\delta(\boldsymbol{l}), \quad \int \boldsymbol{t}^{l} \psi(\boldsymbol{t}) d \boldsymbol{t}=0
$$

for $\boldsymbol{l} \in \mathbb{Z}^{2}, 0 \leq l_{1} \leq L-1,0 \leq l_{2} \leq L-1$, and $l_{1}+l_{2} \leq L-1$, where $\delta(\boldsymbol{l})$ denotes Kronecker delta symbol and $\boldsymbol{t}^{l}$ denotes $t_{1}^{l_{1}} t_{2}^{l_{2}}$. 
2. All moments up to order $(L-1)$ of the lowpass and highpass filters vanish, that is

$$
\sum_{n} n^{l} h(n)=\delta(l), \quad \sum_{n} n^{l} g(n)=0
$$

for $\boldsymbol{l}, l_{1}$, and $l_{2}$ as above, where $\boldsymbol{n}^{l}$ denotes $n_{1}^{l_{1}} n_{2}^{l_{2}}$.

3. The frequency response of the lowpass filter has a zero of order $L$ at the origin and the aliasing frequency $\boldsymbol{\pi}$, that is

$$
\left.\frac{\partial^{l_{1}+l_{2}} H\left(\omega_{1}, \omega_{2}\right)}{\partial \omega_{1}^{l_{1}} \partial \omega_{2}^{l_{2}}}\right|_{\boldsymbol{\omega}=\mathbf{0}, \boldsymbol{\pi}}=0
$$

for $l_{1}$ and $l_{2}$ as above.

Note that the corresponding dual scaling function and wavelet may have different numbers of vanishing moments. The last two sets of conditions provide a useful characterization of BQCW systems, which may be used to construct the associated dual filters.

Since the BCW systems have many advantages over the BSW systems, it is natural to expect the $2 \mathrm{D}$ quincunx extension of the $\mathrm{BCWs}$ to be superior to that of the BSWs. BQCWs are constructed from their 1D counterparts using the McClellan transformation. The frequency response of the synthesis lowpass filter in the $m$ th-order $\mathrm{BCW}$ system may be rewritten as [6]

$$
H_{m}(\omega)=\frac{1}{2}+\sum_{k=1}^{m / 2} 2 h(2 k-1) T_{2 k-1}[\cos \omega] .
$$

where $T_{n}[\cdot]$ denotes the $n$ th-order Chebyshev polynomial [2]. Then, the $2 \mathrm{D}$ frequency response is

$$
H_{m}(\boldsymbol{\omega})=\frac{1}{2}+\sum_{k=1}^{m / 2} 2 h(2 k-1) T_{2 k-1}[F(\boldsymbol{\omega})]
$$

where we have chosen the transformation function to be $F(\boldsymbol{\omega})=\left(\cos \omega_{1}+\cos \omega_{2}\right) / 2$. The same transformation is applied to the dual filter $\widetilde{H}_{m, m^{\prime}}(\boldsymbol{\omega})$. It can be easily shown that such a simple transformation not only allows horizontal, vertical, and diagonal directions to be symmetry axes so that the $2 \mathrm{D}$ frequency response has a diamond-shaped passband, but also preserves some of the properties possessed by $1 \mathrm{D}$ BCW systems such as PR, the number of multiple zeros at the origin and the aliasing frequency, and dyadic fractional filter coefficients. Therefore, this new class of wavelets is promising in image and multidimensional signal processing applications.

\subsection{A design example}

We now demonstrate an example of designing BQCW FBs. The synthesis filter of the 4th-order $\mathrm{BCW}, h_{4}(n)$, is given by

$$
\frac{1}{32}\left[\begin{array}{lllllll}
-1 & 0 & 9 & 16 & 9 & 0 & -1
\end{array}\right] .
$$

One of its associated analysis filters, $\widetilde{h}_{4,4}(n)$, is given by

$$
\frac{1}{512}\left[\begin{array}{lllllllll}
-1 & 0 & 18 & -16 & -63 & 144 & 348 & 144 & \cdots
\end{array}\right]
$$

where the omitted coefficients may be obtained by symmetry. After transformation, we obtain the dual filters of the 4th-order BQCW, $h_{4}(\boldsymbol{n})$ and $\widetilde{h}_{4,4}(\boldsymbol{n})$, which have diamond-shaped spatial supports and are respectively given by

$$
\frac{1}{256}\left[\begin{array}{rrrrrrr} 
& & & -1 & & & \\
& -3 & -3 & 0 & -3 & & \\
& -3 & 0 & 0 & 0 & -3 & \\
-1 & 0 & 39 & 128 & 39 & 0 & -1 \\
& -3 & 0 & 39 & 0 & -3 & \\
& & -3 & 0 & -3 & &
\end{array}\right]
$$

and

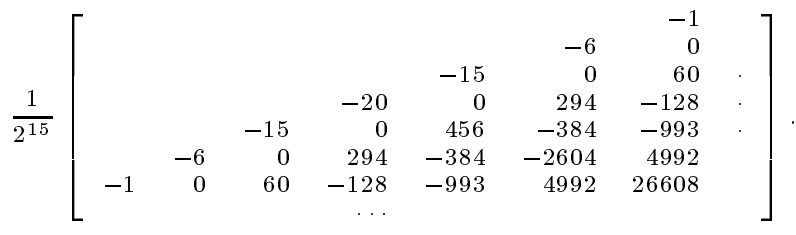

Note that all the filter coefficients are dyadic fractions. Figure 3(a) and Figure 3(b) plot the frequency responses of $h_{4}(\boldsymbol{n})$ and $\widetilde{h}_{4,4}(\boldsymbol{n})$, respectively. We notice the flatness at both the origin and the aliasing frequencies.

\subsection{Asymptotic convergence}

Recently, the asymptotic convergence of the $\mathrm{BCW}$ filters was addressed in [8]. By asymptote we mean that the order of a wavelet system approaches infinity. Now, we extend the $1 \mathrm{D}$ results in [8] to the $2 \mathrm{D}$ case; i.e., we study the asymptotic convergence of the BQCW filters.

Theorem 2. The frequency responses of the $B Q C W$ dual filters converge pointwise to the ideal diamond-shaped halfband lowpass filters as their orders tend to infinity; i.e.,

$$
\begin{gathered}
\lim _{m \rightarrow \infty} H_{m}(\boldsymbol{\omega})= \begin{cases}1 & \text { if }\left|\omega_{1}\right|+\left|\omega_{2}\right|<\pi \\
1 / 2 & \text { if }\left|\omega_{1}\right|+\left|\omega_{2}\right|=\pi \\
0 & \text { otherwise; }\end{cases} \\
\lim _{m, m^{\prime} \rightarrow \infty} \widetilde{H}_{m, m^{\prime}}(\boldsymbol{\omega})= \begin{cases}1 & \text { if }\left|\omega_{1}\right|+\left|\omega_{2}\right| \leq \pi \\
0 & \text { otherwise; }\end{cases}
\end{gathered}
$$


furthermore, the convergence of $H_{m}(\boldsymbol{\omega})$ is monotonic in the sense that,

$$
\begin{array}{ll}
H_{m}(\boldsymbol{\omega}) \leq H_{m+1}(\boldsymbol{\omega}) & \text { if }\left|\omega_{1}\right|+\left|\omega_{2}\right| \leq \pi \\
H_{m}(\boldsymbol{\omega}) \geq H_{m+1}(\boldsymbol{\omega}) & \text { if }\left|\omega_{1}\right|+\left|\omega_{2}\right|>\pi
\end{array}
$$

The above theorem states that the BQCW filters may be viewed as low-order approximations of the ideal diamond-shaped halfband lowpass filters.

\subsection{Interpolating scaling functions}

The synthesis scaling function in a $\mathrm{BCW}$ system is interpolating. It can be shown that after transformation, the resulting $2 \mathrm{D}$ synthesis scaling function is also interpolating; i.e., the interpolating property is invariant to the aforementioned transformation.

Theorem 3. The synthesis scaling function in a $B Q C W$ system of any order is interpolating; i.e., for any $\boldsymbol{n} \in \mathbb{Z}^{2}$,

$$
\left.\phi(\boldsymbol{n})=\delta_{(} \boldsymbol{n}\right)
$$

\section{Summary}

We have presented a new class of compactly supported biorthogonal quincunx wavelets, which possess many interesting and useful properties and are promising in image and multidimensional signal processing. In fact, the proposed results can be easily extended to higher dimensions, e.g., in the case of a face centered orthorhombic (FCO) lattice.

\section{References}

[1] S. Mallat, "A theory for multiscale signal decomposition: the wavelet representation", IEEE Trans. Pattern Anal. Machine Intell., vol. 11, no. 7, pp. 674-693, July 1989.

[2] D. E. Dudgeon and R. M. Mersereau, Multidimensional Digital Signal Processing, Englewood Cliffs, NJ: Prentice-Hall, 1983.

[3] J. Kovačević and M. Vetterli, "Nonseparable multidimensional perfect reconstruction filter banks and wavelet bases for $R^{n}$ ", IEEE Trans. Inform. Theory, vol. 38, no. 2, pp. 533-555, Mar. 1992.

[4] A. Cohen and I. Daubechies, "Non-separable bidimensional wavelets bases", Revista Matemática Iberoamericana, vol. 9, no. 1, pp. 51-137, 1993.

[5] W. Sweldens, "The lifting scheme: a custom-design construction of biorthogonal wavelets", Appl. Comput. Harmon. Anal., vol. 3, pp. 186-200, 1996.
[6] D. Wei, J. Tian, R. O. Wells, Jr., and C. S. Burrus, "A new class of biorthogonal wavelet systems for image transform coding", IEEE Trans. Image Processing, to appear, 1997.

[7] A. Cohen, I. Daubechies, and J.-C. Feauveau, "Biorthogonal bases of compactly supported wavelets", Commun. Pure Appl. Math., vol. 45, pp. 485-560, 1992.

[8] D. Wei and A. C. Bovik, "On asymptotic convergence of the dual filters associated with two families of biorthogonal wavelets", IEEE Trans. Signal Processing, to appear, 1997.

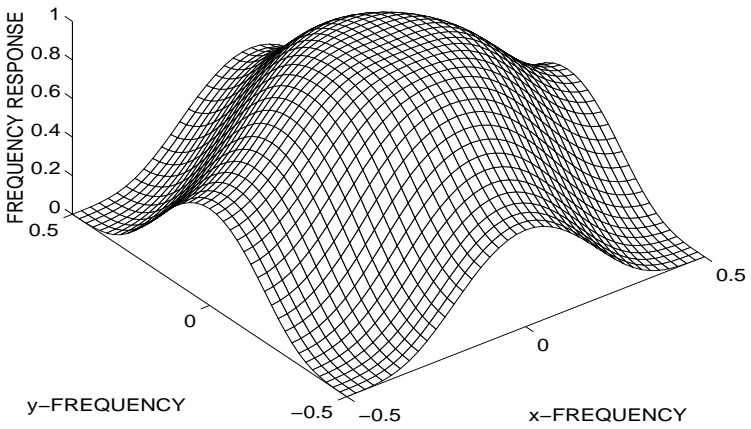

(a)

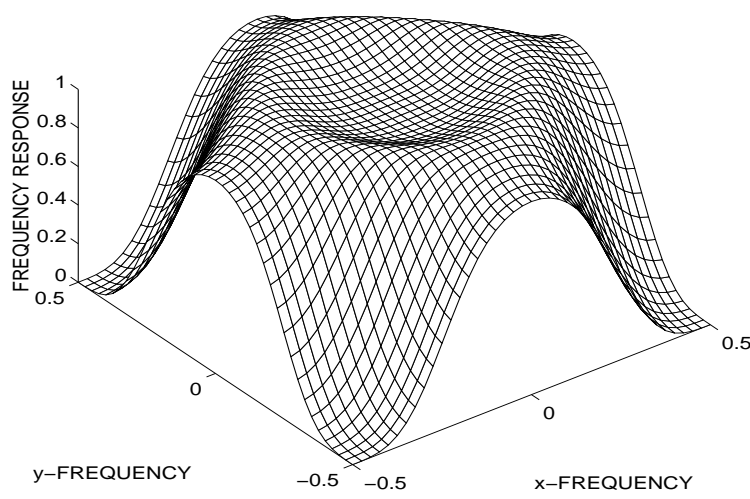

(b)

Figure 3: A design example of BQCW filters 